\title{
A Mathematical Model for Particle Size Distribution in Bell-type Charging at Blast Furnace Top
}

\author{
Takanobu INADA, Yoshimasa KAJIWARA and Tsutomu TANAKA
}

Iron and Steel Research Laboratories, Research and Development Division, Sumitomo Metal Industries, Ltd., Sunayama, Hasaki-cho, Kashima-gun, Ibaraki-ken, 314-02 Japan.

(Received on December 20, 1988; accepted in the final form on April 14, 1989)

\begin{abstract}
A mathematical model for particle size distribution in bell-type charging at blast furnace top was developed, which is capable of estimating the radial average size distribution as well as the radial deposit distribution of each particle size for multiple size burden with a few fixed parameters.

The following findings were considered in the construction of the model. The radial deposit distribution of particles in blast furnace was determined by both the sieving process and the deposit process on the slope. The elemental process in the sieving process is the percolation of small particles through voids between large particles acting as sieves. Principal factors affecting the percolation are the ratio of large particle size to small particle size and the particle velocity gradient in the flowing layer on the slope.

The validity of the model was confirmed through actual filling tests at various blast furnaces with different throat diameters and charging conditions.

The model was applied to the prediction of the change of particle size distribution caused by the decrease of large bell stroke at Wakayama \#4 BF. The monitored results showed the decrease of gas flow resistance and the suppression of peripheral gas flow as were predicted by the model with help of a gas flow simulation model.
\end{abstract}

KEY WORDS: ironmaking; blast furnace process; modeling; simulation; percolation; particle size distribution.

\section{Introduction}

In a blast furnace operation, for an appropriate control of momentum transfer, heat transfer and chemical reactions, the control of burden distribution at the top of blast furnace is as important as that of blasting condition at tuyeres.

As items of the control of burden distribution, the radial distribution of particle size has recently attracted attention in addition to that of ore to coke weight ratio and the separate charge of different particle size burden ${ }^{1,2)}$ and the control of large bell stroke $^{3,4)}$ have been developed.

Consequently, the decrease of the lower size limit of burden has been performed to decrease the pig iron cost. However, at the same time, the increase of the size range of burden brought a new task for the improvement of the estimation accuracy of particle size distribution in blast furnace.

Previous researches on the estimation of the radial distribution of particle size were mainly performed through experiments using either reduced scale models ${ }^{1,3)}$ or full scale models. ${ }^{4}$ Through model experiments, the effects of charging rate, amount of charge and stock level on radial distribution of particle size have been investigated. However, these experiments were performed under specified charging conditions and the applicability of the obtained data was limited.

A trial of measurement of the radial distribution of particle size in operating blast furnaces was attempted by the use of an optical fiber probe installed above the deposit burden at the top of a blast furnace. ${ }^{5)}$ Although there exist restrictions in the surface layer measurement as well as in the scarceness of the measurement frequency, the obtained data provided qualitative knowledge on the radial distribution of particle size in the actual blast furnace.

In order to make more general estimation of the radial distribution of particle size, a few researches by the use of mathematical model have been reported..$^{3,6,7)}$ However, in the one-particle motion model ${ }^{3)}$ the lack of the consideration of the interaction between particles was a remained problem, while in the screening theory model, ${ }^{6)}$ the lack of consideration of the charging rate was a remained problem. In the previous paper, 7) a mathematical model based on the equations of motion on distinct particles and the constitutive equation described by a Voigt-Kelvin rheological model with a slider to simulate interaction between particles was applied for the strict estimation of the radial distribution of particle size for binary size system. Although the effect of the interaction between particles as well as that of charging rate can be considered in the model, the radial distribution of particle size for multiple size system can not be estimated due to extremely large calculation time by computer.

Therefore, in the present research, with consideration of the sieving process and the deposit behavior of particles on the slope, a mathematical model with a 
few fixed parameters was developed for the estimation of the radial distribution of particle size for multiple size system and was applied to an actual blast furnace operation,

\section{Outline of the Mathematical Model}

In the construction of the model for the radial distribution of particle size, the followings were considered.

(1) Concerning the interactions between particles, which were not considered in the one-particle motion model ${ }^{3)}$ the sieving process by the percolation of smaller particles through near-by existing larger particles was considered.

(2) Although the effect of charging rate was not directly considered in the screening theory model, ${ }^{6}$ all of charging conditions and inner state conditions affecting the radial distribution of particle size were considered in the model.

(3) Not only the radial distribution of the average particle size but also the deposit distribution of each particle size for multiple size system can be estimated under the actual charging condition.

(4) The calculation time should be decreased under the condition that the estimation accuracy of the radial distribution of particle size was sufficient.

In Sec. 2.1, the detailed investigation on the sieving process on the slope and the selection of important factors dominating the radial distribution of particle size will be described. Then, the deposit behavior of particles on the slope assumed in the model will be explained in Sec. 2.2. Finally, based on these knowledge, the calculation flow of the mathematical simulation model and the selection of model parameters will be described in Sec. 2.3.

\subsection{Formulation of Sieving Process on the Slope}

Fig. 1 shows the schematic flow behavior of the mixture of large and small particles on the slope. A small particle of diameter $d_{p}$ is flowing down on the slope with large particles of diameter of $d_{b}$. The local velocity of particles and the thickness of the flowing particle layer are designated as $v$ and $h$, respectively.

The elemental process of sieving on the slope is the percolation of the small particle through large particles. The small particle passes through the void

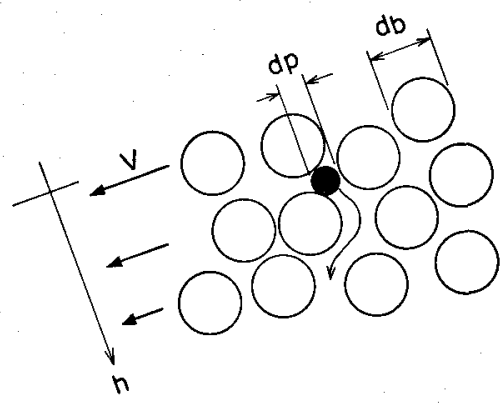

Fig. 1. Schematic flow behavior of mixture of large and small particles on the slope. between large particles, which act as sieves, to reach the lower layer. The percolation frequency of the small particle is dominated by the following two factors.

1) Particle size ratio: $d_{b} / d_{p}(-)$

2) Velocity gradient in the flowing particle layer: $d v / d h(1 / \mathrm{s})$

The former is the ratio of the size of a sieve opening to the size of the particle to be sieved. This value is related to the probability of sieving, when a particle reaches near a sieve opening. The latter is related to the probability of the encounter of the small particle with sieve openings.

Therefore, for particles of the same physical properties, the percolation frequency $(\lambda)$ of a small particle is expressed by Eq. (1).

$$
\lambda=\lambda\left(d_{b} / d_{p}, d v / d h\right)
$$

where, $\lambda()$ : a function.

The dimensionless form of Eq. (1) is expressed by Eq. (2).

$$
\lambda /(d v / d h)=f\left(d_{b} / d_{p}\right)
$$

where, $f(\quad)$ : a function.

Next, the functional formula for $f$ will be determined. Fig. 2 shows the schematic explanation of the possibility of the percolation of a small particle. In Fig. $2(a)$, the sieve opening volume of the lower layer designated as dotted circle is larger than the volume of the small particle to be sieved and the percolation of the small particle into the lower layer is performed. On the other hand, in Fig. 2(b), the sieve opening volume is smaller than the volume of the small particle to be sieved and the small particle is subject to recoil from the lower layer particles to fail in the percolation. Therefore, the possibility of percolation in case of the encounter of the small particle with sieve opening is proportional to the ratio of the free space formed by large particles acting as sieves, which is almost proportional to the volume of large particles, to the volume of the small particle to be sieved.

For the investigation of the appropriateness of this presumption, an experimental result performed by Bridgewater $e t$ al. ${ }^{81}$ is introduced. The experimental apparatus used by them is schematically shown in Fig. 3. Firstly large particles are rectangularly packed in a box. After that, the shear strain with steady rate (w) is introduced into packed bed of large particles by

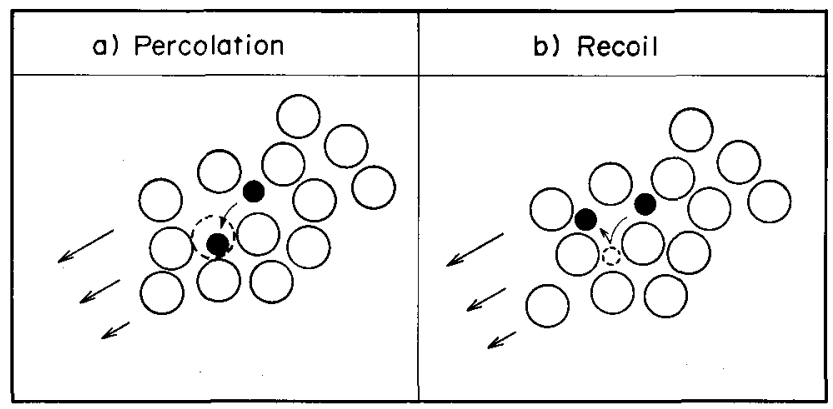

Fig. 2. Scheme of percolation and recoil of a small particle on the slope. 


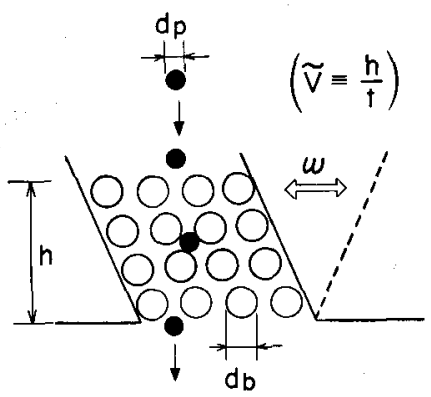

Fig. 3. Experiment of interparticle percolation.

means of reciprocal motion of the side walls of the box. Small particles are inserted one by one at the top of the bed and the residence time $(t)$ of each small particle which descends in the bed by percolation and is discharged from the bottom of the bed is measured. Finally the average descent velocity of small particles $(\tilde{y})$ is evaluated from the residence time $(t)$ and the bed height $(h)$. The ratio of the average descent velocity of small particles in the bed (v) to diameter of large particles $\left(d_{b}\right)$ corresponds to the percolation frequency $(\lambda)$, while strain rate $(\omega)$ corresponds to the velocity gradient $(d v / d h)$. Therefore, $\tilde{v} / v \cdot d_{b}$, that is $\lambda /(d v / d h)$, is plotted against particle size ratio $d_{b} / d_{p}$ in Fig. 4. From the figure, $\lambda /(d v / d h)$ is found to be proportional to $\left(d_{b} / d_{p}\right)^{3}$.

Therefore, Eq. (2) is rewritten as Eq. (3).

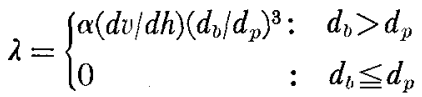

where, $\alpha$ : constant dependent on particle surface properties.

Next, the percolation process in particle stream of binary size system will be explained. Since the total transitions of $n$ small particles during $t \mathrm{~s}$ is expressed by $n \lambda t$, the probability of $i$ time transitions of one small particle $(P(i))$ is expressed as Eq. (4).

$$
P(i)={ }_{n \lambda t} C_{i}(1 / n)^{i}(1-1 / n)^{n \lambda t-i}
$$

When the number of small particles $(n)$ becomes sufficiently large, the probability distribution is expressed by a Poisson distribution.

$$
P(i)=\exp (-\lambda t) \cdot(\lambda t)^{i} / i !
$$

Since a small particle passes through $i$ layers of large particles by the $i$-th percolation in $t \mathrm{~s}$, the descent distance of the small particle $(l)$ is expressed by Eq. (6).

$$
l=i \cdot d_{b}
$$

Accordingly, the probability of small particles existed at the distance $l$ and at the time $t \mathrm{~s}$ is expressed by Eq. (7).

$$
P(l, t)=\exp (-\lambda t) \cdot \frac{(\lambda t)^{l / d_{b}}}{\left(l / d_{b}\right) !}
$$

Fig. 5 shows the distribution of small particles after $t \mathrm{~s}$, which were originally located at $l=0$.

Finally, the particle size distribution after $t \mathrm{~s}$ for

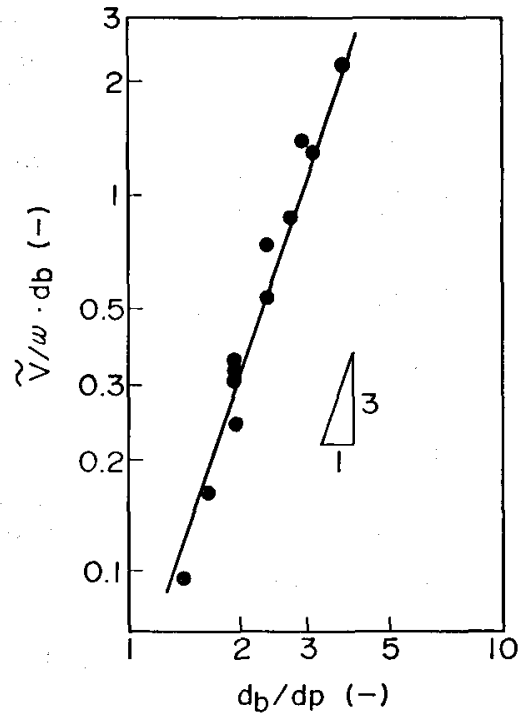

Fig. 4. Relationship between percolation frequency and the ratio of large particle diameter to small particle diameter.

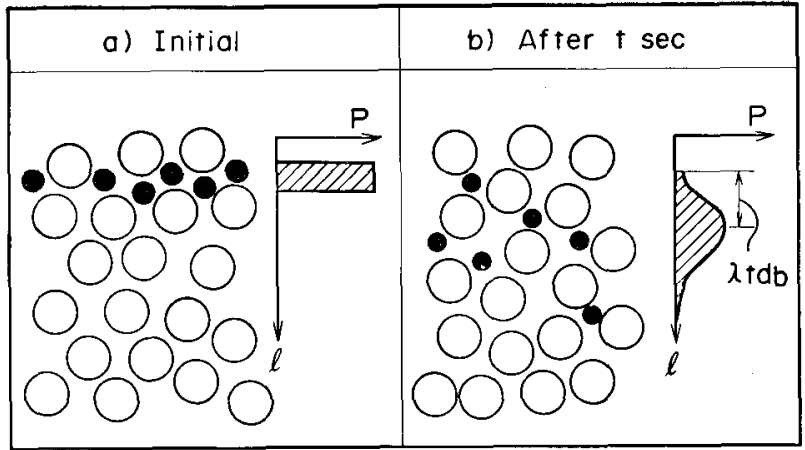

Fig. 5. Distribution of small particles at the initial time and after $l \mathrm{~s}$.

multiple size system will be introduced. In the multiple size system, an intermediate size particle acts both as a particle to be sieved by large particles and as a sieve for small particles. Consequently, Eq. (2) is extended to Eq. (8), when sizes of particles acting for sieving small particle of diameter $d$ are denoted as $d_{b 1}, d_{b 2}, \ldots$

$$
\frac{\lambda\left(d, d_{b i}\right)}{(d v / d h)}=f\left(\frac{d_{b 1}}{d}, \frac{d_{b 2}}{d}, \ldots \ldots\right)
$$

With the assumption that the probability of the encounter of particle of size $d$ with a sieving particle of size $d_{b i}$ is proportional to the average weight fraction of particle of size $d_{b i}$ at the local position $\left(X\left(d_{b i}\right)\right)$, $\mathrm{Eq}$. (8) is rewritten as Eq. (9).

$$
\frac{\lambda\left(d, d_{b i}\right)}{(d v / d h)}=\sum_{d_{b i}>d}\left[X\left(d_{b i}\right) \cdot f\left(\frac{d_{b i}}{d}\right)\right]
$$

By the substitution of Eq. (3) into Eq. (9), the following equation (10) is obtained.

$$
\frac{\lambda\left(d, d_{b_{i}}\right)}{(d v / d h)}=\alpha \sum_{d_{b i}>d}\left[X\left(d_{b i}\right) \cdot\left(\frac{d_{b i}}{d}\right)^{3}\right]
$$


As a result, the probability of particle of size $d$ in the particle flow at the distance $l$ after $t \mathrm{~s}$ for multiple size system becomes Eq. (11).

$$
P_{d}(l, t)=\exp \left[-\lambda\left(d, d_{b i}\right) t\right] \cdot \frac{\left(\lambda\left(d, d_{b i}\right) t\right)^{l / \bar{d}_{b}}}{\left(l / \bar{d}_{b}\right) !}
$$

where, $\bar{d}_{b}=\frac{\sum_{i}\left[d_{b i} \cdot X\left(d_{b i}\right)\right]}{\sum_{i} \overline{X\left(d_{b i}\right)}}$

\subsection{Deposit Behavior of Particles on the Slope}

For the estimation of the radial distribution of particle size, the deposit process of particles on the slope must be considered in addition to the sieving process. Although only a few researches have been reported on the deposit process of small particles on the slope, the importance of the thickness of the flowing particle layer has been clarified. ${ }^{7}$ In case of thick flowing particle layer, even if the sieving rate of small particles is constant, the deposit of particles is completed before the completion of the sieving to result in the suppression of the radial distribution of particle size and vice versa in case of thin flowing particle layer. Therefore, the evaluation of the thickness of the flowing particle layer is very important to improve the estimation accuracy.

\subsubsection{Calculation of Layer Thickness of Particle} Flow on the Slope

Fig. 6 shows the notation used for the calculation of layer thickness of particle flow at the falling point of burden $\left(h_{0}\right) . \quad h_{0}$ is calculated by Eq. (13) under the condition that the charging rate of burden from the bell is equal to the efflux rate of burden to the slope.

$$
h_{0} \simeq \dot{W} /\left(2 \pi R V_{0} \cos \theta \cdot \rho_{b}\right)
$$

where, $R$ : horizontal distance between the falling point and the center of blast furnace (m)

$V_{0}$ : average velocity of burden $(\mathrm{m} / \mathrm{s})$

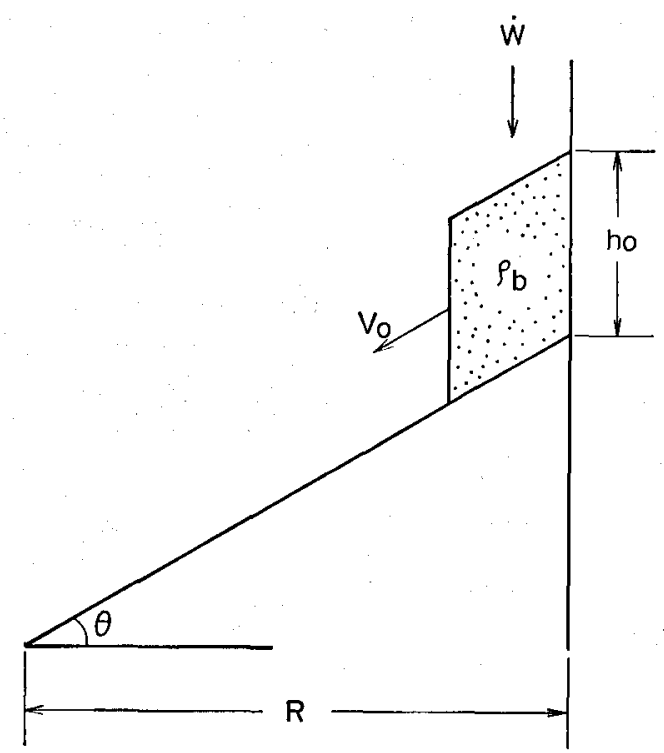

Fig. 6. Notation used for calculation of $h_{0}$.
$\dot{W}$ : charging rate of burden $(\mathrm{kg} / \mathrm{s})$

$\theta:$ angle of slope $\left({ }^{\circ}\right)$

$\rho_{b}$ : bulk density of particle flow $\left(\mathrm{kg} / \mathrm{m}^{3} \mathrm{bed}\right)$. $\dot{W}$ was calculated by the use of the burden discharge model from large bell proposed by Nishio and Ariyama. ${ }^{9} \quad V_{0}$ was evaluated from the calculated free fall velocity with consideration of energy loss at the collision against slope.

The vertical division number of the flowing layer at the falling point $(\mathcal{N})$ was determined as Eq. (14) such that a calculation mesh size is equal to the vertical descent distance of a particle caused by one transition, which correspond to an average particle size of burden.

$$
\mathcal{N}=\frac{h_{0}}{\bar{d}_{b}}
$$

The horizontal division number was set to be equal to the vertical division number $\mathcal{N}$.

\subsubsection{Galculation of Particle Movement in Particle Flow}

The particle movement in the flowing particle layer is schematically shown in Fig. 7. The velocity gradient in the direction perpendicular to particle flow is assumed to be constant and is given by Eq. (15).

$$
\frac{d v}{d h}=\frac{V_{s}^{0}}{h_{0}}
$$

where, $V_{s}^{0}$ : particle velocity at the surface layer of burden at the falling point from bell $(\mathrm{m} / \mathrm{s})$.

The movement of particles between vertical zones in the $i$-th region in $\Delta t \mathrm{~s}$ is calculated as the summation of the small particle movement and the large particle movement caused by sieving.

(1) Movement of Small Particles

Since a particle of size $k$ in $m$-th zone is sieved by a

$$
\begin{array}{ll}
\text { Velocity } & \text { Arrangement } \\
\text { Distribution } & \text { of Particles }
\end{array}
$$

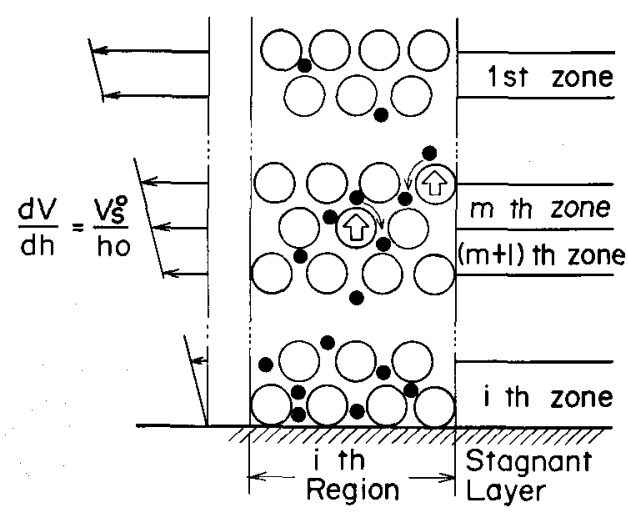

\begin{tabular}{|c|c|}
\hline Key & \multicolumn{1}{|c|}{ Meaning } \\
\hline$\downarrow$ & Percolation of Smal Particles \\
\hline$仓$ & Recoil of Large Particles \\
\hline
\end{tabular}

Fig. 7. Percolation of small particles and recoil of large particles in the $i$-th region on the slope. 
larger particle of size $k^{\prime}\left(k^{\prime}>k\right)$ in the lower zone, the amount of particle of size $k$ moved from $m$-th zone to the lower $m^{\prime}$-th zone through sieving during $\Delta t \mathrm{~s}$, in which particles flow from $(i+1)$-th region to $i$-th region, $\left(\Delta\left(m^{\prime}, m, k\right)\right)$ is expressed by Eq. (16)

$$
\begin{array}{r}
\Delta\left(m^{\prime}, m, k\right)=W(m) \cdot \exp [-\Lambda(k) \Delta t] \cdot \frac{(\Lambda(k) \Delta t)^{\left(m^{\prime}-m\right)}}{\left(m^{\prime}-m\right) !} \\
\ldots \ldots \ldots \ldots \ldots \ldots(\ldots) \\
\Lambda(k)=\sum_{k^{\prime}>k}\left[\lambda\left(k, k^{\prime}\right) \cdot X\left(m, k^{\prime}\right)\right] \\
\lambda\left(k, k^{\prime}\right)=\alpha(d v / d h)\left(k^{\prime} / k\right)^{3} .
\end{array}
$$

where, $W(m)$ : amount of particles in $m$-th zone $(\mathrm{kg})$

$X\left(m, k^{\prime}\right)$ : weight fraction of particles of size $k^{\prime}$ in $m$-th zone (一).

As for the amount of the particle size $k$ which moves from $m$-th zone to the bottom zone (i), it is evaluated as Eq. (19) based on material balance.

$$
\Delta(i, m, k)=W(m) \cdot X(m, k)-\sum_{i>m^{\prime}>m} S\left(m^{\prime}, m, k\right)
$$

(2) Movement of Large Particles

Since the material balance in each zone is held, large particles move upwards when small particles move downwards through sieving.

The amount of particles of size $k$ in $m$-th zone which moves upwards to $(m-1)$-th zone is expressed by Eq. (20).

$$
\begin{aligned}
& \Delta W_{T}(m, k) \\
& \quad=\sum_{k^{\prime}>k}\left[\Delta W_{p}\left(m, k^{\prime}\right) \cdot \frac{\lambda\left(k^{\prime}, k\right) \cdot X(m, k)}{\sum_{k^{\prime}>k^{\prime \prime}}\left(\lambda\left(k^{\prime}, k^{\prime \prime}\right) \cdot X\left(m, k^{\prime \prime}\right)\right\}}\right]
\end{aligned}
$$

where, $\Delta W_{p}\left(m, k^{\prime}\right)$ : amount of particles of size $k^{\prime}$ which move into $m$-th zone from upper zones through sieving

$$
=\sum_{m^{\prime}<m} \sum_{m^{\prime \prime}>m} A\left(m^{\prime \prime}, m^{\prime}, k^{\prime}\right)
$$

Finally, the size composition at $m$-th zone after $\Delta t \mathrm{~s}\left(X^{\prime}(m, k)\right)$ is expressed by Eq. (22) considering both small particle movement and large particle movement caused by sieving.

$$
\begin{aligned}
& X^{\prime}(m, k)=\left\{W(m) X(m, k)+\Delta W_{\text {in }}(m, k)-\Delta W_{\text {out }}(m, k)\right. \\
& \left.+\Delta W_{r}(m+1, k)-\Delta W_{r}(m, k)\right\} /\left[\sum_{j}\{W(m) X(m, j)\right. \\
& +\Delta W_{\mathrm{in}}(m, j)-\Delta W_{\text {out }}(m, j)+\Delta W_{r}(m+1, j) \\
& \left.\left.-\Delta W_{r}(m, j)\right\}\right]
\end{aligned}
$$

where, $\Delta W_{\text {in }}(m, k)=\sum_{m^{\prime}<m} \Delta\left(m, m^{\prime}, k\right)$

$$
\Delta W_{\text {ont }}(m, k)=\sum_{m^{\prime}>m} \Delta\left(m^{\prime}, m, k\right)
$$

2.2.3. Deposit Behavior of Flowing Particle on the Slope

Fig. 8 schematically shows the deposit behavior of flowing particles on the slope. The layer thickness of flowing particles is maximum at the falling point and gradually decreases towards center associated

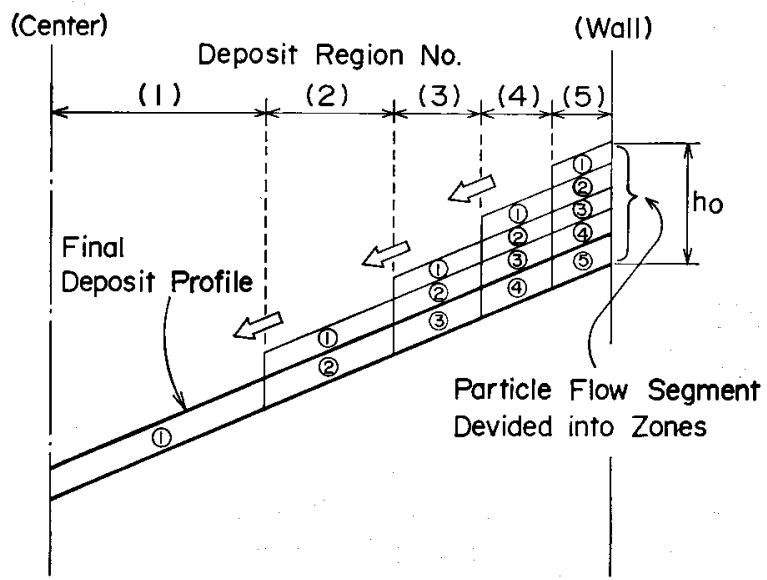

Fig. 8. Deposit behavior of flowing particle on the slope.

with successive deposition of the bottom layer of the flowing particle layer. In the model, for brevity, the amount of deposition at each region is evaluated from the difference between final and initial deposit profile.

Concerning the deposition of particles in the regions located between the falling point and the furnace wall, which appear in the use of movable armor, similar deposit behavior as well as percolation process is assumed. The total amount of burden to be deposited in these regions is calculated from the difference between final and initial deposit profile.

\subsection{Calculation Flow of the Mathematical Model for Par- ticle Size Distribution}

The calculation flow of the model developed with consideration described in Secs. 2.1 and 2.2 is shown in Fig. 9. The layer thickness of particle flow at the falling point of burden $\left(h_{0}\right)$ is calculated by Eq. (13) and the vertical division number of the flowing layer at the falling point $(\mathcal{N})$ is calculated by Eq. (14). The number of radial deposit region is set equal to $\mathcal{N}$. The radial length in each region is determined such that the amount of burden to be deposited in each region is the same.

$\Delta t$ is the traveling time of particles from one deposit region to the next.

As for the initial particle size distribution in each zone of particle flow at the falling point, the comparison of the size composition of deposit particles around the falling point with that of charged particles indicates the occurrence of a certain percolation process in the landing of particles on the precharged burden surface. Therefore it is assumed that "prepercolation" is proceeded in the charged particles before starting to flow down on the slope. The prepercolation time $\Delta t_{0}$ is calculated by Eq. (25) with use of constant parameter $\gamma$ which is evaluated as 0.1 based on the observed size composition of deposit particles at the falling point.

$$
\Delta t_{0}=\gamma \cdot R / V_{0}
$$

Model parameter used in the model is $\alpha$ in Eq. (3). $\quad \alpha_{s}$ for ore and coke were determined with basis of filling results obtained at Wakayama \#3 BF such 
that the difference between the calculated results and the observed results becomes minimum. Sampling of particles was performed by the similar method proposed by Yamada et al. ${ }^{10)}$ The particle size composition of charged burden and the charging condition are shown in Tables 1 and 2, respectively. $\alpha_{s}$ were determined as follows.

$$
\begin{aligned}
\alpha_{\text {ore }} & =0.004 \\
\alpha_{\text {coke }} & =0.006
\end{aligned}
$$

Fig. 10 shows the calculated results using the determined $\alpha_{s}$ in comparison with the observed results. In case of sinter, the deposit fraction of particles of $-20 \mathrm{~mm}$ size is large at the periphery, while it is small at the center. Particle of $-20 \mathrm{~mm}$ size functions as small particle in the sieving process on the slope. In case of coke, particles of $-50 \mathrm{~mm}$ size shows the similar tendency and the threshold size for large and small particles is large for coke than that for sinter. In addition, the decrease of deposit frac-

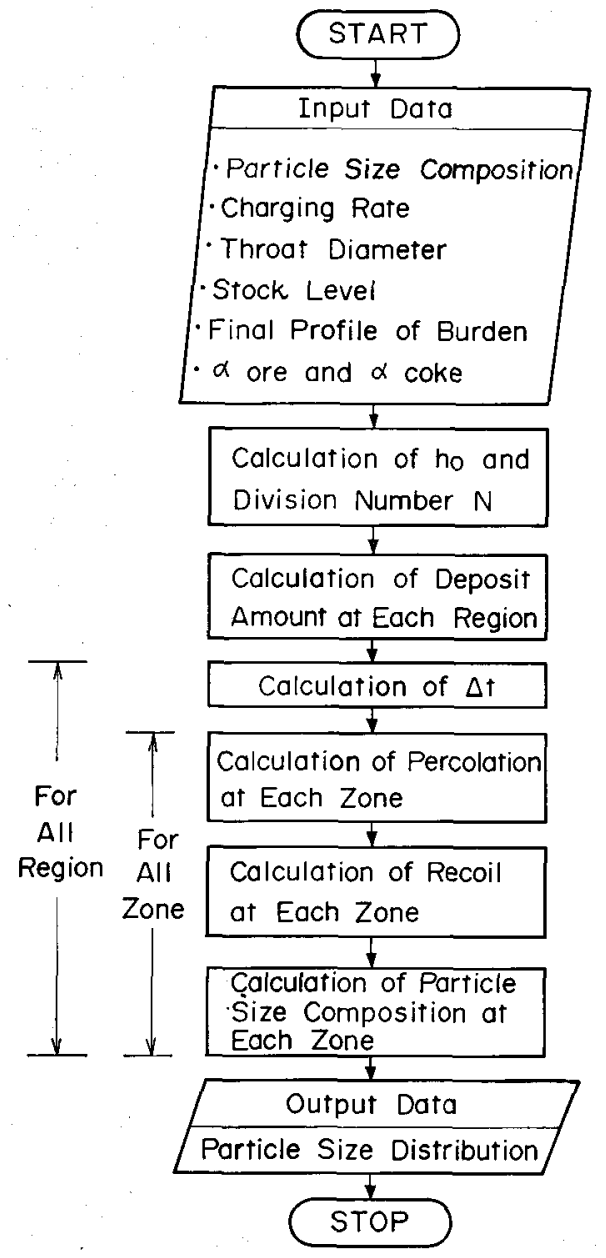

Fig. 9. Calculation flow chart of the mathematical model for particle size distribution. tion of small particles from intermediate region to central region for sinter is more significant than that for coke. This means that the sieving on the slope for sinter particles is enhanced than that for coke particles. The radial distribution of weight average particle size shown in Fig. 11 also reveals the significant sieving in case of sinter. In the figure, M.A. is the abbreviation of movable armor and $\mathrm{C}_{m} \mathrm{O}_{n}$ designates movable armor notch (in Fig. $11, m=0$ and $n=0$ ).

These tendencies of the radial particle size distribution are accurately simulated by the present model with use of a few fixed model parameters of $\alpha_{s}$.

Concerning the division range of the size composition of charged burden as input data for the mathematical model, it is found to be desirable in respect of the simulation precision to divide particle size such that each divided size range contains less than 30 $w \mathrm{t} \%$ of total burden.

\section{Validity of the Mathematical Model for Particle Size Distribution}

The calculated results with use of determined $\alpha_{s}$ regarding the radial size distributions of sinter for different throat diameters and charging conditions (amount of charge and movable armor operation) are compared with measured ones obtained in the filling tests as shown in Fig. 12. The particle size composition of charged sinter was expressed with the same size range for all cases as Table 1. The symbols used in the figure are the same as used in Figs. 10 and 11. At Kashima \#1 BF, large particles segregate in the central region and small particles segregate in the peripheral region because of the enhancement of sieving on the slope caused by the large throat diameter and the small amount of charge in one dump out of 4 dumps in one charge. On the contrary, at Kokura \#2 BF and Wakayama \#4 BF, the segregation of large particles in the central region is not so significant, since the sieving on the slope is not sufficient because of small throat diameters.

At all blast furnaces, small particles segregate at about 0.8 dimensionless radial position which corresponds to the falling point in case of the use of movable armor for sinter. These calculated tendencies are in good agreement with measured ones.

Table 2. Gharging condition in the measurement of particle size distribution at Wakayama \#3 $\mathrm{BF}$.

\begin{tabular}{ll}
\hline Coke base & $15.0 \mathrm{t}$ \\
Charged amount of ore & $52.5 \mathrm{t}$ \\
Sinter ratio & $100 \%$ \\
Movable armor notch & $\mathrm{C}_{0} \downarrow \mathrm{O}_{0} \downarrow$ \\
Stock level & $-3.0 \mathrm{~m}$ \\
\hline
\end{tabular}

Table 1. Particle size composition of charged burden at Wakayama \#3 BF. (wt\%)

\begin{tabular}{lcccrrrrrrrr}
\hline Size $(\mathrm{mm})$ & -5 & $5 \sim 10$ & $10 \sim 15$ & $15 \sim 20$ & $20 \sim 25$ & $25 \sim 30$ & $30 \sim 40$ & $40 \sim 50$ & $50 \sim 60$ & $60 \sim 75$ & +75 \\
\hline Sinter & 2.5 & 28.7 & 21.8 & 13.2 & 9.1 & 6.8 & 9.2 & 2.5 & 3.0 & 1.7 & 1.5 \\
Coke & - & - & - & 0.6 & 2.3 & 6.9 & 13.5 & 23.7 & 21.0 & 25.8 & 9.2 \\
\hline
\end{tabular}



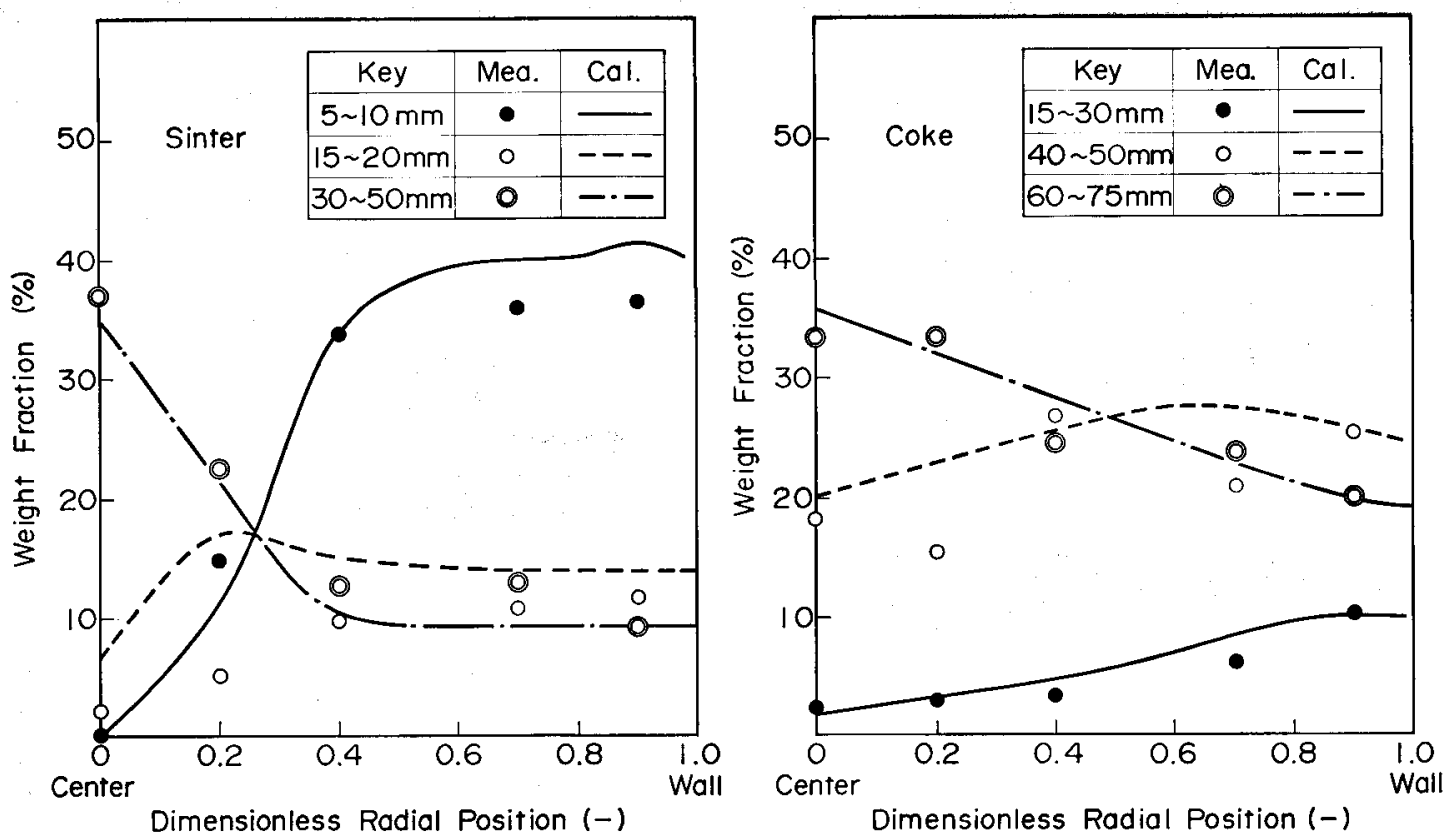

Fig. 10. Comparison of the calculated results of the deposit distribution of each particle size with measured ones at Wakayama \#3 BF.
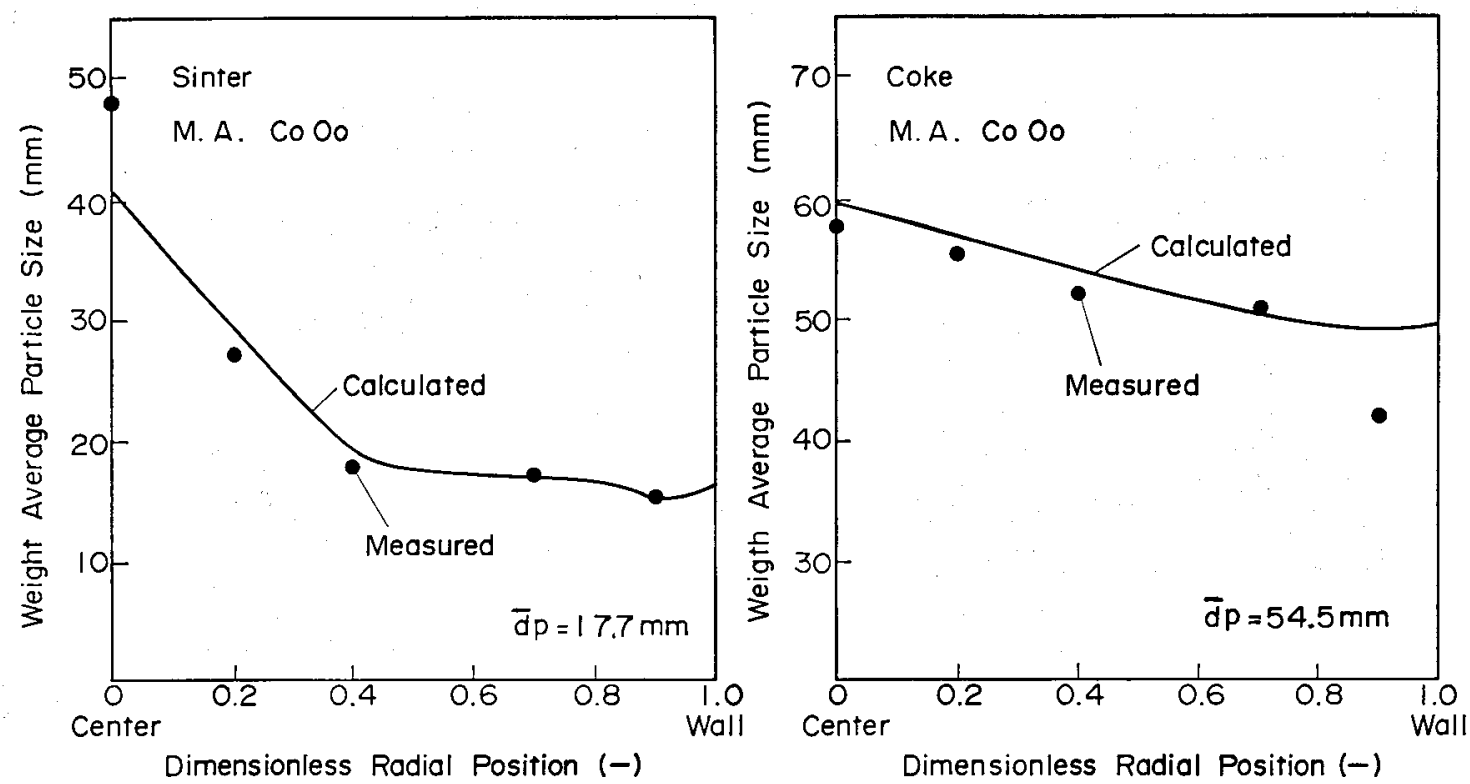

Fig. 11. Comparison of the calculated results of the weight average particle size distribution with measured ones at Wakayama \#3 BF.

\section{Application of the Mathematical Model to Large Bell Stroke Control Test in Actual Blast Furnace Operation}

The decrease of large bell stroke has been developed to reduce gas flow resistance through the enhancement of sieving of small particles on the slope caused by the decrease of charging rate of burden. ${ }^{3}$ )

At Wakayama \#4 BF, the effect of the decrease of large bell stroke on both the gas pressure drop and the change of the radial gas flow distribution was tested. The charging condition is summarized in Table 3. The change of discharging time of burden from large bell with change of large bell stroke was estimated by the use of the discharge model proposed by Nishio and Ariyama') as shown in Fig. 13. The restriction of the discharging time of burden from large bell is dominated by the maximum allowable discharging time required from charging sequence rather than by the hanging limit of coke which was estimated by 5 times of maximum coke size. Accordingly, the large bell stroke was set at $470 \mathrm{~mm}$ in the test operation.

The changes of radial weight average size distribution and gas velocity distribution through the change of large bell stroke estimated by the model with help of gas flow mathematical mode ${ }^{111}$ are shown in Figs. 14 and 15, respectively. It is predicted that the segregation of small particles in the peripheral region is enhanced by the decrease of large bell stroke and 


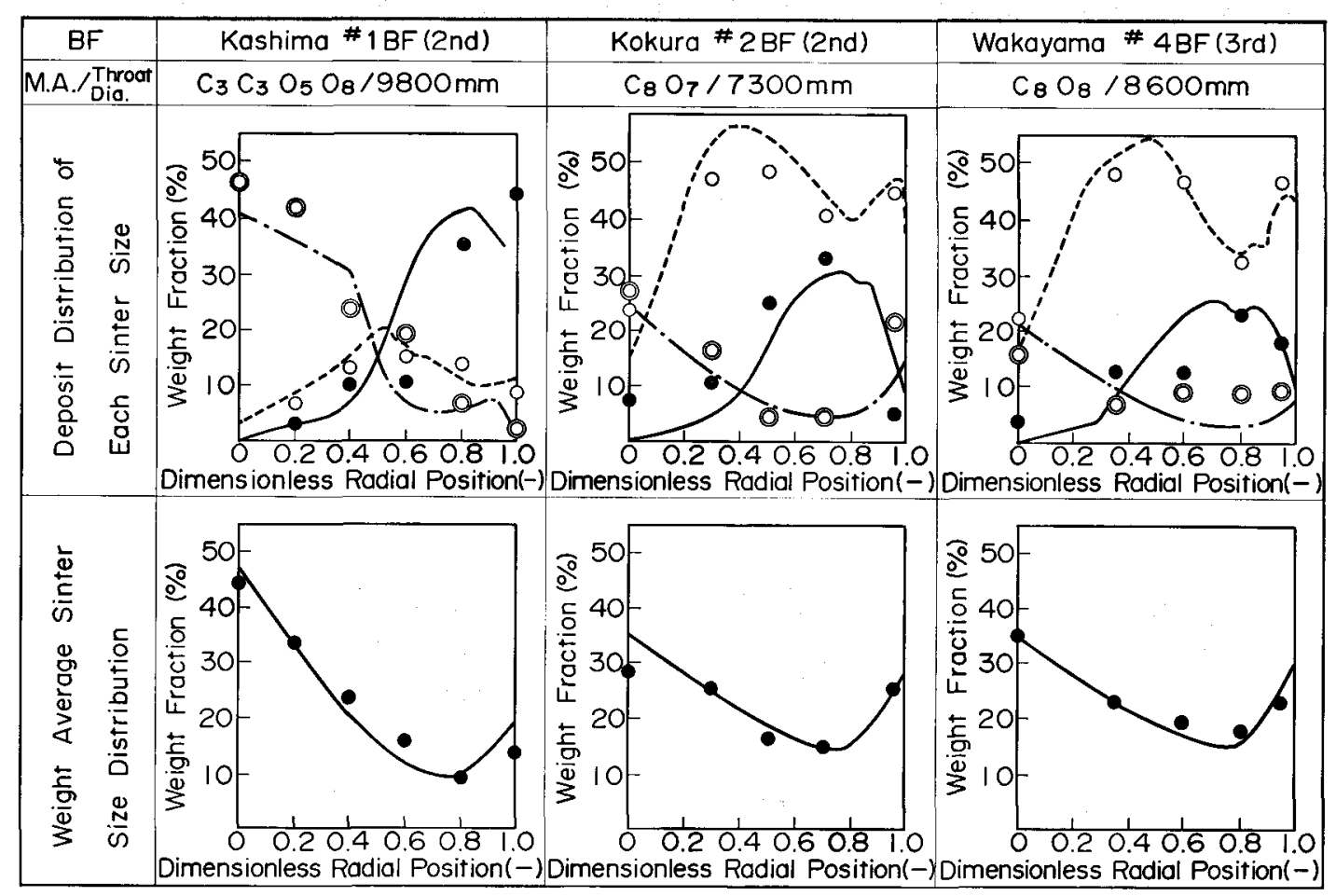

Fig. 12. Comparison of the calculated results of the deposit distribution of each sinter size and weight average sinter size distribution with measured ones for various blast furnaces.

Table 3. Charging condition in the test operation for large bell stroke change at Wakayama \#4 BF.

\begin{tabular}{ll} 
Coke base & $16.0 \mathrm{t}$ \\
Charged amount of ore & $56.5 \mathrm{t}$ \\
Sinter ratio & $80 \%$ \\
Movable armor notch & $\mathrm{C}_{2} \downarrow \mathrm{O}_{0} \downarrow$ \\
Stock level & $-3.0 \mathrm{~m}$ \\
Large bell stroke & $650,470 \mathrm{~mm}$ \\
Large bell descent speed & $72 \mathrm{~mm} / \mathrm{s}$ \\
\hline
\end{tabular}

causes the decrease of gas velocity in the peripheral region and the increase of gas velocity in the central region.

The operational result at Wakayama \#4 BF from January to February, 1987 is shown in Fig. 16. As was expected, the decrease of large bell stroke caused the reduction of gas flow resistance indicated by $K r$ index. ${ }^{12)}$ As clear from the definition of $K r$ index described as Eq. (26), the decrease of $K r$ index indicates the reduction of gas flow resistance caused by the change of the burden packing condition, which actually is the change of the radial particle size distribution.

$$
K r=\frac{(\Delta P / L)}{(215 / 0.027 \mu)^{\beta} \rho_{1 /}^{1-\beta} u^{2-\beta}}
$$

where, $L$ : layer height $(\mathrm{m})$

$\Delta P:$ total pressure drop in blast furnace $(\mathrm{kg} /$ $\mathrm{m} \cdot \mathrm{s}^{2}$ )

$u:$ gas velocity (average) $(\mathrm{m} / \mathrm{s})$

$\beta:$ constant parameter $(-)$

$\mu:$ gas viscosity (average) $(\mathrm{kg} / \mathrm{m} \cdot \mathrm{s})$

$\rho_{g}:$ gas density (average) $\left(\mathrm{kg} / \mathrm{m}^{3}\right)$.

Further, the suppression of peripheral gas flow was

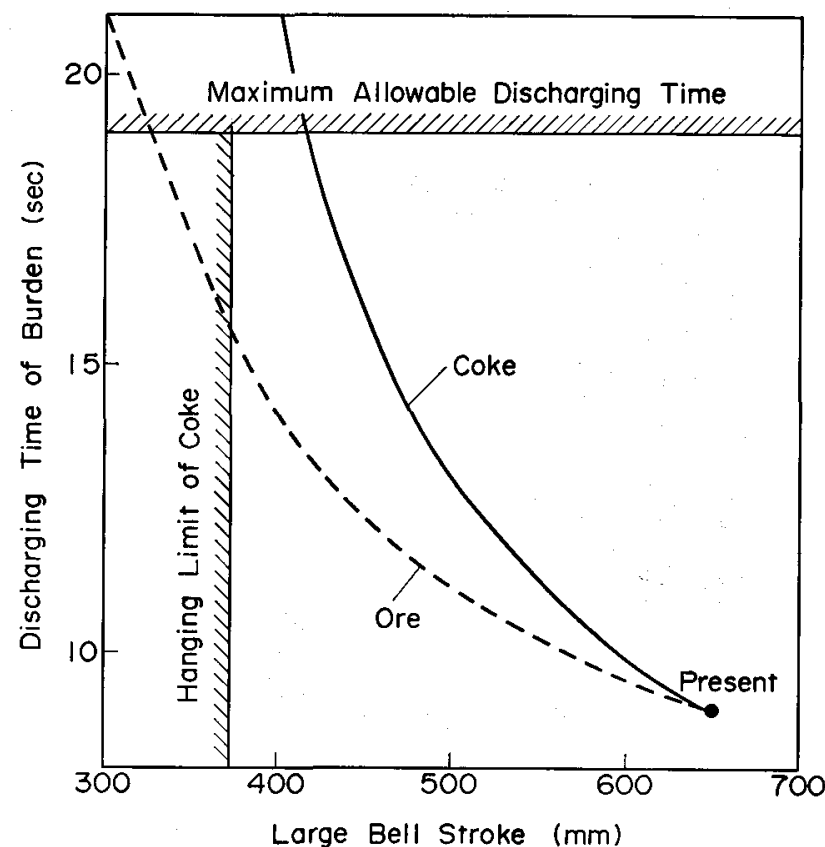

Fig. 13. Change of discharging time with change of large bell stroke at Wakayama \#4 BF.

observed through monitoring by skin flow temperatures and stave temperatures.

\section{Discussion}

\subsection{Restriction of the Application of the Mathematical Model}

In the present model, percolation of small particle is implicitly assumed. Therefore, the model is not applicable to flowing particles in thin layer thickness. 


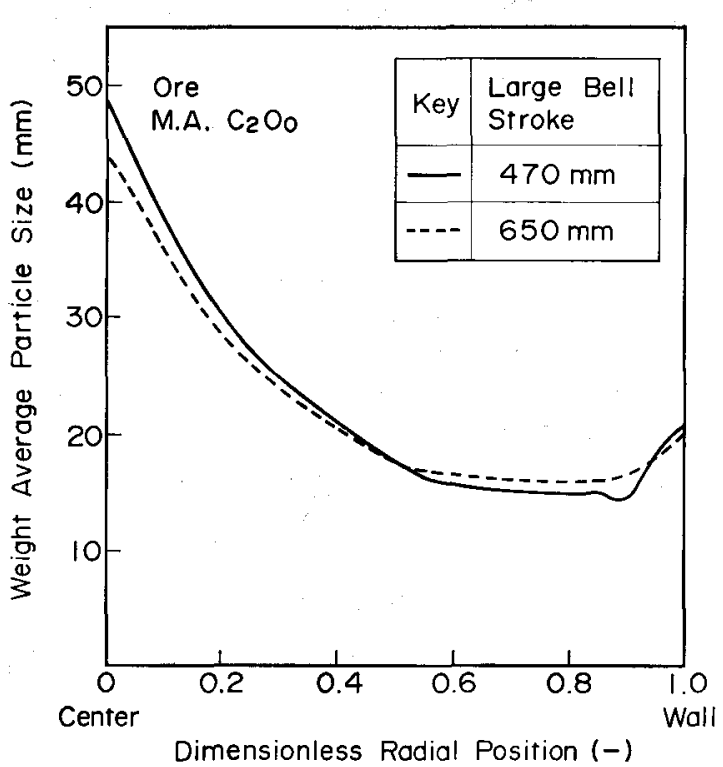

Fig. 14. Effect of large bell stroke on the radial distribution of weight average particle size at Wakayama \#4 $\mathrm{BF}$.

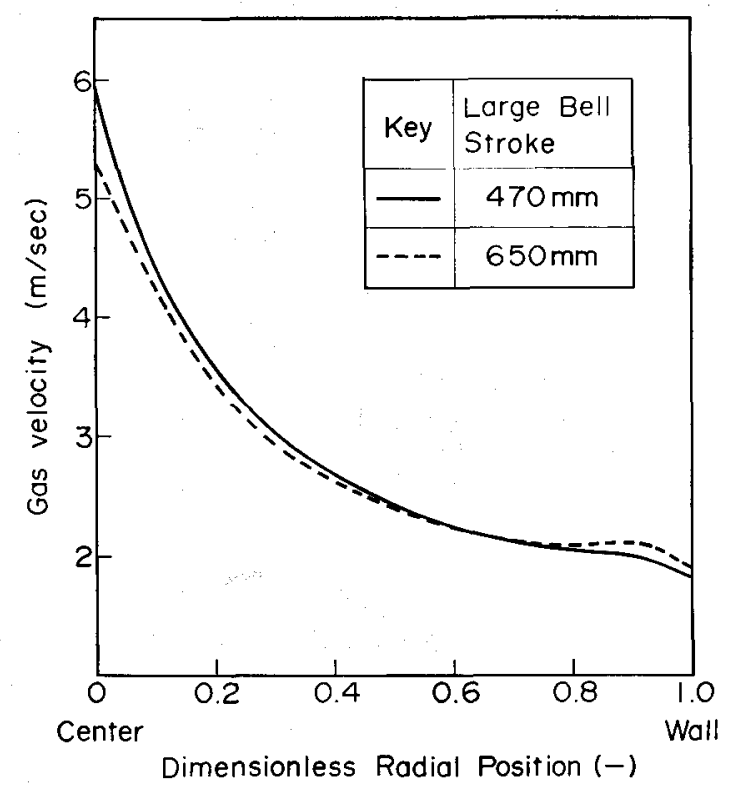

Fig. 15. Effect of large bell stroke on the radial distribution of gas vclocity at Wakayama \#4 BF.

Suppose the division number over 4 is required for sufficient percolation. Then, the model can be applied only to the following charging condition.

$$
\mathcal{N}=\frac{h_{0}}{\tilde{d}_{b}}=\frac{\dot{W}}{2 \pi R V_{0} \cos \theta \cdot \rho_{b} \bar{d}_{b}}>4
$$

where, $\mathcal{N}$ : vertical division number $(-)$.

Eq. (27) is obtaincd with use of Eq. (13).

Inserting typical values for ore $\left(\rho_{b}=1800 \mathrm{~kg} / \mathrm{m}^{3}\right.$ and $\left.d_{b}=0.020 \mathrm{~m}\right)$, for coke $\left(\rho_{b}=500 \mathrm{~kg} / \mathrm{m}^{3}\right.$ and $d_{b}=$ $0.040 \mathrm{~m})$ and for angle of slope $\left(=30^{\circ}\right)$, the applicable conditions are $\dot{W} / R V_{0}>784\left(\mathrm{~kg} / \mathrm{m}^{2}\right)$ for ore and $\dot{W} / R V_{0}>435\left(\mathrm{~kg} / \mathrm{m}^{2}\right)$ for coke. These conditions are satisfied in all bell-type charging, while they are not satisfied in bell-less charging because of low $\dot{W}$ value.

Accordingly the model can only to the bell-type

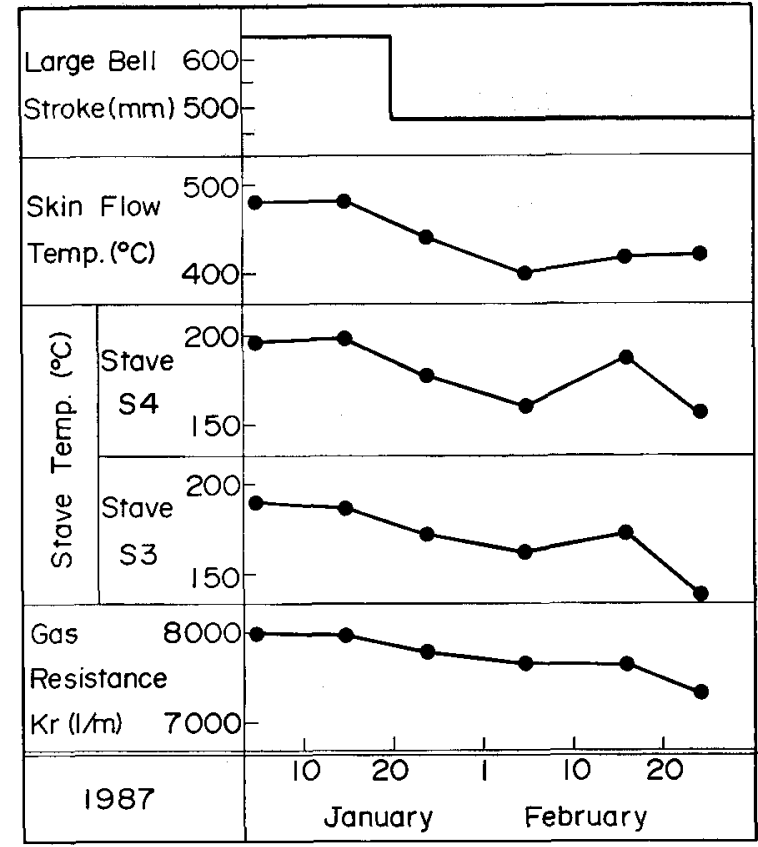

Fig. 16. Operational result by the change of large bell stroke at Wakayama \#4 BF.

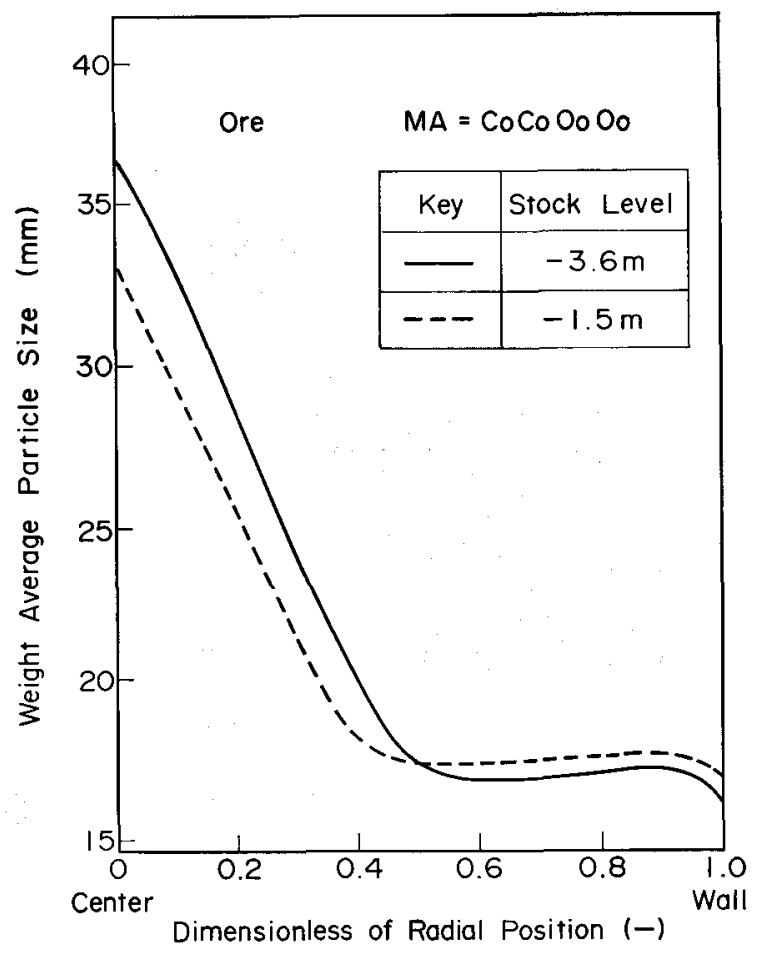

Fig. 17. Effect of stock level on the radial distribution of weight average particle size at Kashima \#1 BF.

charging. This fact is reflected to the title of this paper.

\subsection{Effect of Stock Level on the Radial Distribution of Particle Size}

The effect of stock level on the radial distribution of particle size was investigated by the model for Kashima \#1 BF as shown in Fig. 17. The enhancement of particle size segregation by the decrease of stock level from stock line -1.5 to $-3.6 \mathrm{~m}$ is consistent with the report in the previous full scale model 


\section{experiments. ${ }^{4}$}

Next, the reason for the enhancement of the size segregation by the decrease of stock level is considered. The lowering of the stock level causes the increase of the falling velocity of burden at the falling point resulting in the increase of the velocity of flowing particles on the slope. As a result, the allowable time for percolation of small particles on the slope is reduced to cause the suppression of the radial size segregation.

On the other hand, the decrease of the stock level causes not only the decrease of the thickness of the flowing particle layer as is expected from Eq. (13) but also the increase of the velocity gradient within the flowing particle layer as is expected from Eq. (15). The former reduces the number of percolations required for small particles to reach the bottom of flowing layer and the latter enhances the percolation of small particles in the flowing layer. Both factors cause the enhancement of the radial segregation.

Accordingly, it is estimated that in the actual blast furnace the effect of factors enhancing the radial size segregation is more significant than that suppressing the radial size segregation. Therefore, the decrease of stock level caused the enhancement of the radial size segregation both in the present model calculation and in the previous model experiment.

\section{Conclusion}

Based on measured results on the radial particle size distribution in the filling test as well as the analysis of the fundamental research on the percolation of small particles, a mathematical model was developed for the estimation of the radial particle size distribution and was successfully applied to the test operation of large bell stroke control at Wakayama \#4 BF. Through the investigation, the following knowledge was obtained.

(1) The radial particle size distribution of particles was dominated both by sieving process and deposit process.

(2) The sieving process is essentially the percola- tion of small particles, which are determined by the particle size composition and the velocity distribution in the flowing layer.

(3) Percolation of multiple size system is expressed by an extended Poisson distribution.

(4) The developed model can be used for estimation of not only the average particle size distribution but also deposit distribution of each particle size.

(5) The model was successfully applied to the prediction of the decrease of gas flow resistance and the suppression of peripheral gas flow caused by the enhancement of size segregation through the decrease of large bell stroke in the test operation at Wakayama \#4 $\mathrm{BF}$.

\section{REFERENCES}

1) Y. Okuno, T. Irita, T. Isoyama, T. Imai, Y. Hara and A. Suzawa: Tetsu-to-Hagané, 69 (1983), 1578.

2) J. Kurihara, H. Takahashi, K. Okumura, S. Takeuchi and Y. Karigome: Tetsu-to-Hagané, 65 (1979), S91.

3) T. Hara, M. Saino, M. Yasuno, K. Okumura, K. Kaneko, Y. Konishi and T. Sawada: Trans. Iron Steel Inst. Jpn., 24 (1984), 482.

4) N. Inagaki, M. Baba, K. Asai, N. Kusakabe and T. Suehiro: Tetsu-to-Hagané, 69 (1983), S56; Trans. Iron Steel Inst. Jpn., 23 (1983), B358.

5) S. Murakawa, S. Taguchi, H. Momokawa, T. Sawada, T. Minagawa and M. Seki: Tetsu-to-Hagané, 72 (1986), S49.

6) M. Kondoh, Y. Konishi, K. Okabe, S. Tomita, S. Hashizume and J. Kurihara: Proc. of Int. Conf. on Operation of the Blast Furnace Theory and Practice (HF80), ArlesFrance, (1980), I-3-1.

7) Y. Kajiwara, T. Inada and T. Tanaka: Trans. Iron Steel Inst. Jpn., 28 (1988), 916.

8) J. Bridgewater, M. H. Cooke and A. M. Scott: Trans. Inst. Chem. Engrs., 56 (1978), 157.

9) H. Nishio and T. Ariyama: Tetsu-to-Hagané, 68 (1982), 2330.

10) T. Yamada, M. Sato, S. Miyazaki, H. Shimamura and S. Taguchi: Kawasaki Steel Tech. Rep., 6 (1974), 16.

11) M. Hatano and K. Kurita: Tetsu-to-Hagané, 62 (1976), 953.

12) H. Matoba and T. Shimoda: Tetsu-to-Hagané, 60 (1974), S354. 